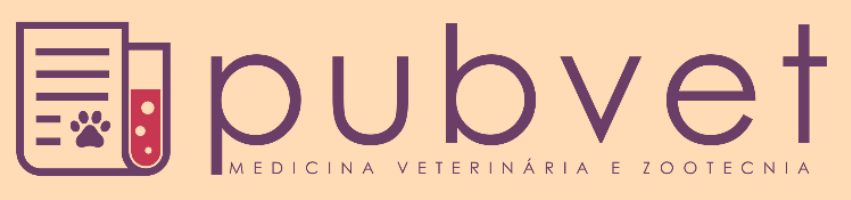

https://doi.org/10.31533/pubvet.v16n02a1045.1-5

\title{
Complexo hiperplasia endometrial cística - piometra em cadela nulípara de 10 meses: Relato de caso
}

\author{
Nathan Feliciano ${ }^{*} \otimes$, Mateus Dozzo Mathias ${ }^{2} \oplus$, Patrick Eugênio Luz $^{3 \oplus}$ \\ ${ }^{1}$ Discente de Medicina Veterinária Unifil, Londrina -PR, Brasil. \\ ${ }^{2}$ Médico Veterinário no Hospital Veterinário Santa Clara Saúde Animal, Londrina-PR, Brasil. \\ ${ }^{3}$ Mestrando do Programa de Pós-graduação em Ciência Animal da Universidade Estadual de Londrina (UEL), e Docente do \\ Departamento de Clínica Médica de Pequenos Animais Universidade Unifil, Londrina - PR, Brasil. \\ *Autor para Correspondência, E-mail: nathan_feliciano@hotmail.com
}

\begin{abstract}
Resumo. A piometra pode acometer mais comumente cadelas de meia idade à animais idosos, ou cadelas jovens que façam uso de hormônios exógenos. Essa doença está relacionada com o diestro, fase na qual ocorre maior estímulo pelo hormônio progesterona com consequente desenvolvimento da hiperplasia endometrial cística e alterações nas funções do útero. Nesse trabalho, relatou-se um evento incomum de piometra aberta em uma cadela de 10 meses, sendo presente apenas a secreção vaginal no exame físico e poucas alterações no exame de sangue que pudessem sugerir um quadro infeccioso. O diagnóstico por imagem foi essencial nesse caso e proporcionou um tratamento adequado para o animal.
\end{abstract}

Palavras-chave: Diestro, hec, útero

\section{Cystic endometrial hyperplasia complex - pyometra in a 10-month- old nulliparous bitch: Case report}

\begin{abstract}
Pyometra can most commonly affect middle-aged bitches to older animals, or young bitches that use exogenous hormones. This disease is related to diestrus, a phase in which there is a greater stimulation of the hormone progesterone and the consequent development of cystic endometrial hyperplasia, with changes in the functions of the uterus. In this study, an unusual event of open pyometra in a 10-month-old dog was reported, with only vaginal secretion present on physical examination and few changes in blood test that could suggest an infectious condition. Imaging diagnosis was essential in this case and provided an appropriate treatment for the animal.
\end{abstract}

Key words: Diestrus, ceh, uterus

\section{Complejo de hiperplasia endometrial quística - piometra en una perra nulípara de 10 meses: Reporte de caso}

Resumen. La piometra puede afectar más comúnmente a las perras de mediana edad que a los animales de edad avanzada, o a las perras jóvenes que utilizan hormonas exógenas. Esta enfermedad está relacionada con el diestro, fase en la que existe una mayor estimulación por parte de la hormona progesterona, con el consecuente desarrollo de hiperplasia endometrial quística y alteraciones en las funciones del útero. En este estudio, se informó un evento inusual de piometra abierta en una perra de 10 meses, con secreciones vaginales presentes en el examen físico y pocos cambios en el análisis de sangre que pudieran sugerir una condición infecciosa. El diagnóstico por imagen fue fundamental en este caso y proporcionó un tratamiento adecuado al animal.

Palabras clave: Diestro, hec, útero 


\section{Introdução}

A piometra é uma infecção aguda ou crônica do útero que está associada ao acúmulo intraluminal de pus logo após o estro de cadelas de meia idade à idade avançada (Evangelista, 2009; Pretzer, 2008). Essa condição, ocorre devido ao aumento do estímulo de progesterona durante a fase de diestro, hormônio esse que desempenha importante papel na etiologia da doença. $\mathrm{O}$ aumento nos níveis de progesterona desencadeado pelo corpo lúteo, exerce efeitos no desenvolvimento da hiperplasia endometrial cística (HEC), com acúmulo de líquido nas glândulas endometriais e lúmen uterino, além de diminuição da contratilidade do miométrio e supressão da atividade imunológica no útero (Barsanti et al., 2004). Unicamente, a HEC não desenvolve sinais clínicos, mas precede o desenvolvimento da infecção. Juntamente com a diminuição da resposta imunológica do útero, predispõe à colonização de bactérias, originando o quadro de piometra (Crane, 2015). Frequentemente, a HEC e piometra são associadas como uma só doença (Evangelista, 2009; Pretzer, 2008). Entretanto, esses dois distúrbios podem ocorrer de forma independente (Hagman, 2018). A bactéria Escherichia coli é o microrganismo isolado mais comumente encontrado na piometra canina (Macphail, 2014). Entretanto, o trabalho realizado por Trautwein et al. (2017) demonstrou que a porcentagem de bactérias E.coli encontrada no conteúdo uterino foi de $18 \%$, ou seja, bem menor do que é relatado por outros autores. A cultura vaginal não é de grande ajuda no diagnóstico, já que a mesma microbiota na piometra é presente em animais saudáveis (Hagman, 2018).

A piometra, pode ter apresentação fechada sem secreção vulvar, ou aberta, com secreção. Os sinais clínicos podem variar, e a forma aberta está relacionada com sinais mais brandos e melhor prognóstico (Barsanti, 2015). Os sinais clínicos apresentam-se dentro de 2 a 4 meses após o estro e incluem descarga vaginal mucopurulenta ou hemorrágica, anorexia, depressão, polidipsia, poliúria, taquicardia, taquipneia, pulso fraco, febre, desidratação, vômito, dor abdominal à palpação, anormalidades de marcha e diarreia (Hagman, 2018).

A incidência para piometra aumenta com o avanço da idade e em cadelas nulíparas (Crane, 2015). Apesar de ser mais comum em cadelas mais velhas, a administração de hormônios exógenos como estrógeno e progesterona, podem levar ao desenvolvimento da doença em cadelas mais novas. A idade média de ocorrência é dos 7,0 aos 8,5 anos, porém, tem-se relatos em cadelas de 4 meses até 18 anos (Evangelista, 2009; Pretzer, 2008).

A piometra é pouco descrita em cadelas mais jovens, por isso, esse trabalho possui o objetivo de relatar um caso de piometra após o primeiro cio de uma cadela, com o intuito de alertar os médicos veterinários sobre sua casuística e melhorar o prognóstico dos pacientes.

\section{Relato de caso}

Foi atendido na Clínica Santa Clara Saúde Animal, Londrina - Paraná, uma paciente canina, raça Buldogue Francês, fêmea, 10 meses de idade, pesando 8,5kg, apresentando queixa de hematúria.

Durante a anamnese, o proprietário relatou que há três semanas havia terminado o primeiro cio da paciente, negando qualquer administração de hormônios exógenos. Durante o exame físico, foi observado sangramento vaginal leve, frequência cardíaca e respiratória de $120 \mathrm{bpm}$ e $32 \mathrm{mpm}$ respectivamente, mucosas normocoradas, tempo de perfusão capilar (TPC) 2 segundos, normohidratado, temperatura retal $38,2^{\circ} \mathrm{C}$ e nenhum sinal de abdominalgia.

Optou-se pela realização de hemograma e bioquímica sérica, onde foi possível observar policromasia, lipemia e diminuição dos valores de alanina-aminotransferase (ALT). Mediante as alterações discretas em exame hematológico, o paciente foi encaminhado para casa sem nenhum tratamento, e orientado a retornar caso o sangramento não cessasse.

Após 17 dias, retornou com a mesma queixa de secreção vulvar. O proprietário alegou que após o primeiro atendimento, seu animal apresentou sangramento apenas por alguns dias e cessou, no entanto, a secreção voltou de forma mais intensa com coloração amarela à marrom. Teve um episódio de vômito em casa, além de fezes mais amolecidas. Ao exame físico foi possível verificar apenas desconforto abdominal e secreção vulvar amarronzada. Mediante alterações observadas durante a avaliação, foi solicitado avaliação ultrassonográfica, onde foi possível visualizar esplenomegalia e o corpo do útero 
com dimensões aumentadas (1,60 cm de diâmetro), parede espessa, mucosa irregular e moderado conteúdo luminal anecogênico. $\mathrm{O}$ paciente foi então submetido à realização de um novo exame de sangue, onde foi revelado discreta anisocitose, leve trombocitopenia 141.000 (175-500.000), hiperproteinemia 7,8 (5,0-7,0) e hipoalbuminemia 1,9 (2,6-3,3).

Frente a tais alterações, optou-se pela internação da paciente, sendo instituído tratamento com solução de Ringer com Lactato como fluidoterapia ( $27 \mathrm{~mL} / \mathrm{hr}$ ), cloridrato de tramadol $3 \mathrm{mg} / \mathrm{kg} \mathrm{IV} \mathrm{a}$ cada oito horas, dipirona $25 \mathrm{mg} / \mathrm{kg}$ IV a cada oito horas, enrofloxacina $10 \mathrm{mg} / \mathrm{kg}$ IV a cada 24 horas e ondansetrona $0,5 \mathrm{mg} / \mathrm{kg}$ IV a cada oito horas, para posterior realização de ovário-histerectomia $(\mathrm{OH})$.

Realizou-se como medicação pré-anestésica (MPA) a combinação de fentanil $3 \mathrm{mcg} / \mathrm{kg}+$ midazolam $0,2 \mathrm{mg} / \mathrm{kg}$ IV. Para a indução anestésica optou-se pela utilização de propofol $3 \mathrm{mg} / \mathrm{kg} \mathrm{IV}$. Durante o procedimento cirúrgico a paciente foi mantida em anestesia inalatória com isoflurano. No período transoperatório foi realizada analgesia com morfina na dose $0,3 \mathrm{mg} / \mathrm{kg}$ IV e aplicação de ampicilina na dose de $30 \mathrm{mg} / \mathrm{kg} \mathrm{IV}$.

Sob efeito da anestesia, a paciente foi posicionada em decúbito dorsal na mesa cirúrgica para realização da antissepsia. Realizou-se incisão pré-retroumbilical em linha média, contemplando pele e subcutâneo, com posterior incisão em estocagem na linha alba para acesso à cavidade abdominal. $\mathrm{O}$ útero repleto foi tracionado com cautela para evitar ruptura, demonstrando estar aumentado (Figura 1).

Após ser evidenciado, o pedículo ovariano direito foi pinçado e ligado com sutura transfixante, circundando de forma simultânea os vasos e ligamento suspensor do ovário. Posteriormente, a mesma técnica foi utilizada no pedículo ovariano esquerdo. No coto uterino, realizou-se ligadura simples individual dos vasos, associada com ligadura transfixante dupla em coto uterino. Ambas as ligaduras foram realizadas com fio não absorvível nylon 2-0. A síntese da cavidade abdominal foi realizada com fio absorvível poliglecaprone 2-0 e recebeu 3 diferentes tipos de sutura. A síntese de musculatura foi realizada com padrão Sultan simples separado, a síntese de subcutâneo foi realizada com padrão Cushing, e a síntese de pele com padrão Wolff. Posteriormente á realização da $\mathrm{OH}$, foi evidenciado conteúdo purulento no interior do útero através da punção com uma seringa (Figura 2).

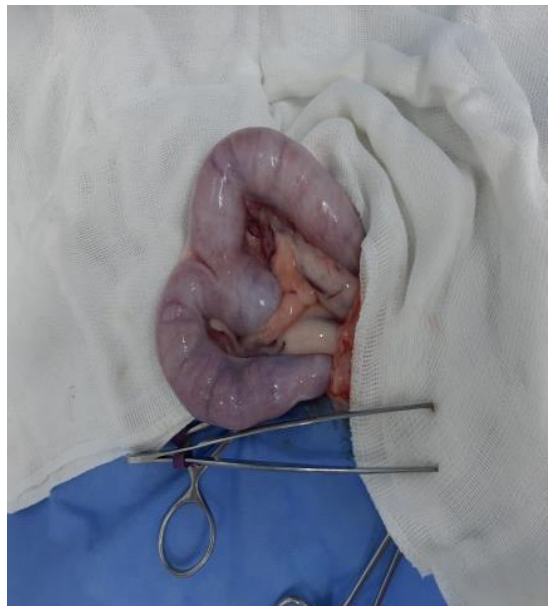

Figura 1. Corpo e cornos uterinos sendo expostos.

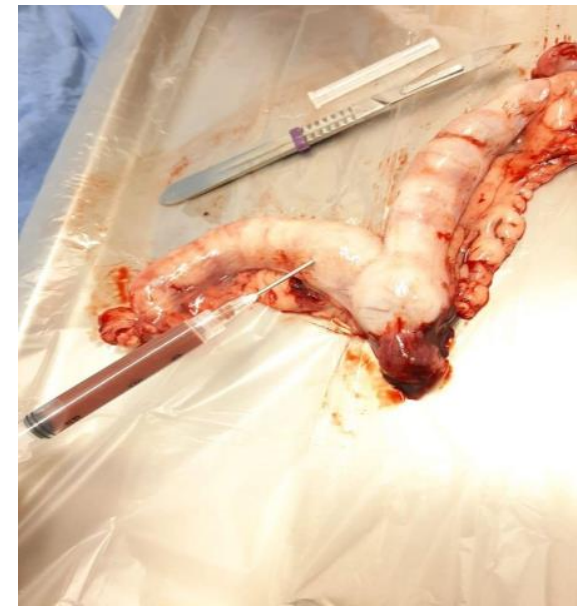

Figura 2. Útero logo após ser retirado, evidenciando conteúdo purulento em seu interior quando submetido à punção com uma seringa.

Após procedimento, a paciente ficou internada por 24 horas, sendo acrescido anti-inflamatório nãoesteroidal (meloxican $0,1 \mathrm{mg} / \mathrm{kg}$ intravenoso) em seu tratamento. Um novo hemograma pós-operatório, realizado no dia seguinte à cirurgia, revelou discreta anemia normocítica normocrômica 39,0\% (40-47), anisocitose e poiquilocitose, leucocitose $27.900 / \mathrm{mm}^{3}(8.000-16.000)$ com neutrofilia $19.809 \%$ (4.50011.200), linfócitos em limite superior 6.417\% (1.600-6.400), além de monocitose 1.674\% (150-1.280) e trombocitopenia $96.000 / \mathrm{mm}^{3}(175.000-500.000)$.

Para o tratamento domiciliar, foi prescrito medicação para proteção do trato gastrintestinal, omeprazol $1 \mathrm{mg} / \mathrm{kg}$ via oral a cada 24 horas por sete dias, além de antibiótico de amplo espectro, 
enrofloxacina $10 \mathrm{mg} / \mathrm{kg}$ via oral a cada 24 horas por sete dias, analgesia com cloridrato de tramadol 2 $\mathrm{mg} / \mathrm{kg}$ via oral a cada oito horas por quatro dias, dipirona uma gota por $\mathrm{kg}$ via oral a cada oito horas por sete dias e anti-inflamatório não-esteroidal meloxican $0,2 \mathrm{mg} / \mathrm{kg}$ via oral a cada 24 horas por dois dias. Sendo solicitado um retorno em 7 dias para reavaliação do paciente e retirada de pontos.

Ao retornar, o proprietário relatou melhora da paciente, sendo repetido exame hematológico para avaliação plaquetária, verificando que estavam dentro da referência $\left(204.000 / \mathrm{mm}^{3}\right)$.

\section{Discussão}

O presente animal desenvolveu piometra com 10 meses, sendo esta uma idade menos frequente. De acordo com o trabalho realizado por Rodrigues (2019), a maioria dos pacientes atendidos desenvolveram piometra dos oito aos 11 anos, representando (36\%) dos casos, seguido por cadelas de quatro a sete anos (30\%), cadelas a partir de 12 anos (20\%) e por fim cadelas de até três anos representando $14 \%$ dos casos. No estudo retrospectivo de Costa et al. (2020) as cadelas foram classificadas e acometidas nas seguintes idades: em menos de três anos (6\%), entre quatro e sete anos (25\%) e a partir dos oito anos (61\%).

A paciente apresentou apenas corrimento vaginal no primeiro atendimento, sem mais sinais que pudessem sugerir um quadro de piometra. Assim como cita (Davidson, 2014), a descarga vaginal mucopurulenta ou hemorrágica pode ser o único sinal em alguns casos. O diagnóstico é realizado com base na apresentação clínica, exame físico e ginecológico, além de exames hematológicos e bioquímicos, ultrassonografia e radiografia. A apresentação clássica da doença com descarga vaginal é mais fácil de diagnosticar quando comparado com a forma fechada, além disso a febre pode não estar presente em metade dos casos (Hagman, 2018), assim como ocorreu nesse paciente.

A ultrassonografia se mostrou de grande importância, sendo um meio indispensável para quadros com suspeita de piometra. É o método de diagnostico mais utilizado e pode evidenciar corpo uterino com conteúdo anecoico ou hipoecóico (Crane, 2015). O tipo de conteúdo luminal também revela achados importantes. De acordo com Hagman (2018), a ultrassonografia possibilita achados como cistos ovarianos, HEC e algumas vezes possibilita a diferenciação da piometra para mucometra ou hidrometra por meio da ecogenicidade do fluido.

O exame de sangue pré-cirúrgico não foi significativo para confirmação da suspeita nesse relato. Achados como anemia normocítica normocrômica regenerativa são comuns (Hagman, 2018), no entanto, não estiveram presentes no exame, assim como não houve leucocitose. A diminuição do hematócrito ocorre pela ausência de resposta da medula frente a eritropoetina, o que é causado pela endotoxemia e sepse que ocorre de forma secundaria á infeção uterina (Sant'Anna et al., 2019).

Não foi observado elevação dos níveis de marcadores renais neste caso, o que é esperado na maioria dos pacientes com tal enfermidade. Em seu estudo, Maddens et al.( 2010) observaram que a infeção causada pela piometra é primordial para a ocorrência da lesão renal, sendo individual a resposta que cada paciente vai reagir a essa agressão. E alguns biomarcadores renais uteis na medicina humana, demonstraram precocidade na identificação da lesão renal, mesmo em pacientes não azotêmicos, que revelaram ser a maioria nesse trabalho.

A ausência de resultados importantes no exame pode ser explicada pelo bom estado geral do paciente. Segundo Macphail (2014), a frequência cardíaca maior que $160 \mathrm{bpm}$, temperatura maior que $39,6^{\circ} \mathrm{C}$ ou menor que $37,7^{\circ} \mathrm{C}$, frequência respiratória maior que $20 \mathrm{mpm}$ ou pressão parcial de $\mathrm{CO}_{2}$ menor que 32 $\mathrm{mmHG}$ e contagem de células brancas maior que $12.000 / \mu \mathrm{L}$, menor que $4.000 / \mu \mathrm{L}$ ou bastonetes acima de $10 \%$, indicam um quadro de síndrome da resposta inflamatória sistêmica (SIRS), isso quando, pelo menos dois dos quatro critérios estiverem presentes no animal. Desta forma, o prognóstico tende a ser pior.

A presença de hiperproteinemia, hiperglobulinemia e hipoalbuminemia foram alguns dos achados que, assim como explica Crane (2015), podem estar associados à desidratação ou sepse. Segundo Li \& Chan (2016), são causas de trombocitopenia: mielossupressão, bem como sequestro esplênico, hemodiluição, trombose intravascular e coagulação intravascular disseminada (CID).

Apesar de ser uma condição de emergência, é importante que o animal seja estabilizado antes de ser encaminhado para o centro cirúrgico (Bocardo et al., 2008; Carvalho et al., 2008; Pretzer, 2008). Assim que internado, o animal recebeu imediatamente fluidoterapia e antibioticoterapia, corroborando com o 
descrito por Barsanti (2015), dando preferência para antibacterianos de amplo espectro eficazes contra a bactéria E.coli, como a enrofloxacina.

\section{Considerações finais}

A piometra pode acometer cadelas jovens, que não foram submetidas ao efeito de hormônios exógenos. Esse relato, mostra que a piometra deve ser considerada em cadelas apresentando secreção vaginal mucóide, hemorrágica ou purulenta, além de outros sinais que podem ser a única forma de identificar a piometra fechada. Independentemente do exame físico, dos exames de sangue e da idade do animal, a piometra sempre deve ser um dos diagnósticos diferenciais. A ultrassonografia se mostrou eficaz, sendo essencial para o diagnóstico definitivo neste paciente. É importante que os pesquisadores realizem mais estudos para entender a fisiopatogenia da doença, e compreender como cadelas mais jovens podem desenvolver a piometra logo após o primeiro cio.

\section{Referências bibliográficas}

Barsanti, J. (2015). Genitourinary nfections. In C. E. Greene (Ed.), Infections dieseases of the dog and cat (pp. 1039-1043). Elsevier Editora.

Barsanti, J. A., Lees, G. E., Willard, M. D., \& Green, R. A. (2004). Urinary disorders. In T. Willard (Ed.), Small animal clinical diagnosis by laboratory methods (Vol. 4, pp. 135-164).

Bocardo, M., Hamzé, A. L., \& Zappa, V. (2008). Piometra: Técnicas cirúrgicas e clínicas para o tratamento. Revista Científica Eletônica de Medicina Veterinária, 11, 1-7.

Carvalho, C. C. D., Rêgo, E. W., Queque, M., \& Soares, P. C. (2008). Avaliação da proteína C reativa, fibrinogenio e leucograma em cadelas com piometra. Medicina Veterinária, 2, 3-10.

Costa, S. P. A., Mariano, D. B., \& Monteiro, R. C. P. (2020). Estudo retroespectivo da casuística de piometra em cadellas atendidas em hospital veterinário escola no período de cinco anos. Revista Saúde-UNG-Ser, 13(2), 81.

Crane, M. B. (2015). Pyometra. Small animal critical care medicine. Elselvier Saunders.

Davidson, A. P. (2014). Female and male infertility and subfertility. Small Animal Internal Medicine, 2, 951-965.

Evangelista, L. S. M. (2009). Alterações clínicas e laboratoriais em cadelas com piometra antes e após ovariossalpingohisterectomia. In Veterinária: Vol. Master of. Universidade Federal de Teresina.

Hagman, R. (2018). Pyometra in small animals. Veterinary Clinics of North America: Small Animal Practice, 48(4), 639-661. https://doi.org/10.1016/j.cvsm.2018.03.001.

Li, R. H. L., \& Chan, D. L. (2016). Evaluation of platelet function using multiple electrode platelet aggregometry in dogs with septic peritonitis. Journal of Veterinary Emergency and Critical Care, 26(5), 630-638. https://doi.org/10.1111/vec.12508.

Macphail, C. M. (2014). Surgery of the integumentary system. In T. W. Fossum (Ed.), Small animal surgery. Elsevier Editora.

Maddens, B., Daminet, S., Smets, P., \& Meyer, E. (2010). Escherichia coli pyometra induces transient glomerular and tubular dysfunction in dogs. Journal of Veterinary Internal Medicine, 24(6), 1263 1270. https://doi.org/10.1111/j.1939-1676.2010.0603.x.

Pretzer, S. D. (2008). Clinical presentation of canine pyometra and mucometra: a review. Theriogenology, 70(3), 359-363.

Rodrigues, R. M. (2019). Padrão etário e racial de doenças reprodutivas em cadelas na região metropolitana de Belém-PA (Issue 47). Universidade Federal Rural da Amazônia.

Sant'Anna, M. C., Martins, G. F., Flaiban, K. K. M. C., Trautwein, L. G. C., \& Martins, M. I. M. (2019). Relação proteína-creatinina-urinária no diagnóstico precoce de lesão renal em cadelas com piometra. Pesquisa Veterinária Brasileira, 39, 186-191. https://doi.org/10.1590/1678-5150-PVB-5624.

Trautwein, L. G. C., Sant'Anna, M. C., Justino, R. C., Giordano, L. G. P., Flaiban, K. K. M. C., \& Martins, M. I. M. (2017). Piometras em cadelas: relação entre o prognóstico clínico e o diagnóstico laboratorial. Ciência Animal Brasileira, 18, 1-4. https://doi.org/10.1590/1089-6891v18e-44302

Histórico do artigo:

Recebido: 5 de outubro de 2021

Aprovado: 1 de novembro de 2021

Disponível online: 28 de fevereiro de 2022
Licenciamento: Este artigo é publicado na modalidade Acesso Aberto sob a licença Creative Commons Atribuição 4.0 (CC-BY 4.0), a qual permite uso irrestrito, distribuição, reprodução em qualquer meio, desde que o autor e a fonte sejam devidamente creditados. 